\title{
All-in-One Companion for Visually Impaired
}

\author{
Love Aggarwal \\ B.Tech (ECE), GGSIPU
}

\author{
Varnika Gaur \\ B.Tech (ECE), GGSIPU
}

\author{
Puneet Verma \\ B.Tech (ECE), GGSIPU
}

\begin{abstract}
:
In this paper, the design of an All-In-One Companion for Visually Impaired has been proposed. The system aims to help such people while they are alone at their homes. The system will carry out the orders given by its user (a visually impaired man/woman) by analyzing his/her voice ${ }^{[1]}$ and also by their hand gestures ${ }^{[2]}$.
\end{abstract}

The system aims to provide necessary features for visually impaired like home automation. In this, different devices like fan, light, etc., which will be already interfaced with the system, can be turned on/off, by making different gestures, with the help of IR sensors mounted on the fingers of the user. RF signals are used for communication between the devices and sensors.

Also, the systems aims to provide other features amongst which the key feature is security of the user. This feature has been proposed keeping in mind that the user is visually impaired. A GSM Modem with a SIM will be activated immediately when the user gives voice command - "HELP" and will send an SMS alert, containing the location of the user as given by the GPS Module, will be sent to all the recipients including local authorities and kin of the user. In addition to it, a buzzer will be activated simultaneously which will generate an alarm to alert anyone nearby.

Other features include two-way communication with the system via GSM Modem, a Gas Sensor to detect fire and a Temperature Sensor to detect the environment surroundings. Thus the system aims to be an ALL-IN-ONE STICK to support visually impaired, speaking figuratively.

Keywords: GPS, GSM, IR Sensors, Gas Sensors, RF Signals, SMS, Two-way Communication

\section{INTRODUCTION}

Eyes are one of the five senses and provide us vision. Everyone learn from what they see and perceive through our eyes. Therefore, without these one cannot imagine a normal life.

Understanding the importance of eyes, one can imagine the life of visually impaired. These people have to do their daily chores without seeing what they are doing or where they are. Thus, the same amount of work done by a visually impaired will take more time and effort as compared to a sighted person. Furthermore, such people require special care as they are more prone to danger. Having a family around will sure benefit visually impaired but not all live in a family, some live alone too. Therefore, for such people, not even their homes are safe. There have been many incidents like thief breaking into their homes etc. Thus, their security in such cases remains a major concern. One approach to resolve this issue is installing cameras in their homes for surveillance. However, this may harm their privacy. Another approach can be building a robot for all their needs but, this may not be cost effective. Therefore, this research paper focusses on the basic necessities and security of such people. Basic necessities have been provided through the home automation feature. Under this feature, few devices such as fan, light etc. can be controlled wirelessly via finger gestures. The security feature is provided by sensors which can detect intruders, smoke, fire etc. Furthermore, SMS alerts can be sent to the concerned people.

Although research can be extensive but practically the system should be feasible, cost effective and reliable. One should believe in systems which are built in public interests and easily accessible. Thus, their cost effectiveness is of utmost importance. Moreover, the system should be easy to install and update. This will ensure that the system works without any hindrance.

\section{RELATED WORK}

A considerable amount of work has been done to provide assistance to the visually - impaired. For example, one such work is a prototype system titled "A Prototype System to Support the KJ Method by Cooperation between visually impaired persons and sighted persons" [3]. It was based on KJ Method. It is an idea generation method, quite popular in Japan. This system was developed to assist visually impaired people in performing the KJ method with the sighted people. It is impossible for such people to perform the KJ method otherwise because of method's requirements. Therefore these researchers proposed the use of three devices: table - type display, Braille PDA and tactic display. Using the prototype system, the visual information can be converted to tactile or auditory information and thus, can assist them in performing this method.

Another example of such work is the system titled "Voice Navigation system for the Visually Impaired by Using IC Tags" ${ }^{[4]}$. In this a white cane system is described that can support the independent working of the visually impaired indoors. The system comprises of mainly two things: colored navigation lines which are put on the floor of the target space from the starting point to end point and an intelligent white cane that has a navigation computer. IC tags are included in the navigation lines and are set at the landmark point. These tags are used to indicate the destination route. Furthermore, the white cane comprises of a color sensor, an IC tag transceiver and a computer system which includes a voice processor. Vibration in the white cane informs the user that he/she is on the navigation line and thus following this vibration, user reaches the destination. Pre-recorded voice is used for communication with the user.

Another example is the prototype titled "Low-cost face biometry for visually impaired users" ${ }^{[5]}$. Its developers have proposed that it can implement the trends of videos analysis and support in improving communication of visually impaired. The prototype processes the incoming video streams and locates the known faces. Thereafter, each event of interest produces an audio feedback for the user. This feedback allows the user to locate the presence of people. 


\section{OUR APPROACH}

This paper mainly focuses on providing home automation and security to the visually impaired. For home automation, few devices are taken to keep the system design simple yet working. This feature will be accessed through finger movement of IR sensors. The security feature comprises of anti-intruder function, fire safety, sensing of environment etc. These will be provided using the IR sensors, gas sensors and temperature sensor. The sensors are cheap and reliable and therefore, are suitable. Also, keeping the disability of the user in mind, communication with the device is done through voice commands. The feature to include all the spoken languages for communication is being considered.

\section{TECHNICAL REQUIREMENTS}

The technical requirements of the system are stated below:

\subsection{Microcontroller}

Atmega328 microcontroller is used to provide the hardware platform for the system. It functions as the brain/controlling unit of the system. All the other components such as sensors, GSM, GPS, RF module, devices (fan etc.) etc. are interfaced with the microcontroller.

\subsection{GSM Modem}

GSM or Global System for Mobile Communication is a standard which is developed to describe protocols for the second generation $(2 \mathrm{G})$ of mobile communication. It is a wireless modem that works on wireless network. The GSM modem is configured using AT commands. GSM has been used in the system for establishing two way communication and to send SMS alerts.

\subsection{GPS Module}

GPS or Global Positioning System is a satellite navigation system. It is capable of providing the location (longitude and latitude) and time information in any weather conditions. GPS has been used in the system to provide the location of the user so that the user can be reached in time of danger.

\subsection{IR Sensor ${ }^{[6]}$}

An Infra-red sensor detects and/or measures the infra-red radiation in the surroundings. These sensors are able to detect the heat and motion of the object. An IR Sensor has two LEDs: Emitter and Detector. Four such IR sensors have been utilized in the system. Out of which three are used to detect the different finger gestures, on which they are mounted. The fourth IR Sensor is installed at the entrance to detect any intrusion.

\subsection{Gas Sensor ${ }^{[7]}$}

A gas sensor detects the presence of combustible or flammable gases. It gives reading in ppm (parts per million). A gas sensor has been used in the system to detect the presence of any harmful gas that can result in fire etc.

\subsection{Temperature Sensor}

A temperature sensor, as the name suggests, measures the temperature or temperature gradient of the surroundings. This sensor has been utilized in the system to provide temperature readings of the surroundings via two way communication.

\subsection{RF Module}

Radio Frequency or RF Module consists of two parts: Transmitter (Tx) and Receiver (Rx). It is available in different operating frequencies with different operating range. An Encoder Circuit and a Decoder Circuit is used along with the Transmitter and Receiver respectively in order to transmit and receive the message/signal ${ }^{[8]}$. In this system the RF module is used to provide the native communication task between the IR sensors \& Home Automation Unit (devices interfaced) and IR sensor (for intrusion) \& Controlling Unit.

\subsection{Voice Recognition IC: APR 9600 ${ }^{[9]}$}

This IC is used for high-quality voice recording \& playback. It supports both random and sequential access of multiple messages. It's easy to use and user friendly. This IC has been utilized in the system to make voice communication possible with the visually impaired user.

\subsection{Buzzer}

A common buzzer of $5 \mathrm{~V}$ has been used to generate alarm in situations where any intruder is detected or a fire breaks out or the user requires any help.

\section{WORKING}

As stated above, this system (block diagram shown in Figure 1) aims to provide home automation and security to the visually impaired. Home automation has been provided by interfacing few devices with IR sensors. These devices are those which are generally required by the visually impaired at home and difficult to access for them. Initially, one can take three basic devices such as: Fan, Light and Radio. For each of the devices, an IR sensor has been mounted on the finger of the user. In other words, each of the device is synchronized with its respective IR sensor. The data provided by the IR sensor is processed by the microcontroller. The communication between the devices and the sensors is done through RF signals ${ }^{[10]}$ When any finger gesture ${ }^{[2]}$ is made by the user, the respective IR sensor gets activated. Then the device, which is synchronized with the respective sensor, is turned ON through RF signals. Any gesture made again now will turn off the device. Thus, the devices can be easily operated (on/off) just by the finger gestures. This will help the visually impaired in accessing the basic necessities at their homes. The block diagram for Home Automation feature is shown in Figure 2. The Linker (Circle, named "A") in Figure 1 and Figure 2 is used to show the connection (flow of signals) between the Main Controlling Unit and the Home Automation Unit. And the Linker (Circle, named "B") shown in Figure 1 and Figure 3 is used to show the connection (flow of signals) between the Main Controlling Unit and the Security Unit.

The system also aims to provide security to the user. In case of an emergency, a GSM modem is used to issue SMS alerts to the local authorities and the kin of the user. Such a situation can arise from intruder detection or a fire alarm or the user requiring help. If, due to any reasons the user feels insecure, then he/she should issue simple voice command "1] "HELLO". This command will be immediately recognized by the ARP 9600 IC and the microcontroller will activate the GSM modem, GPS and buzzer. The buzzer will start sounding alarm immediately to alert anyone nearby. The GPS module will provide the location details of the user. Finally, the GSM will issue SMS alerts containing the location and help appeal. The recipient contact numbers can be entered in the code prior to the installation of the system. 


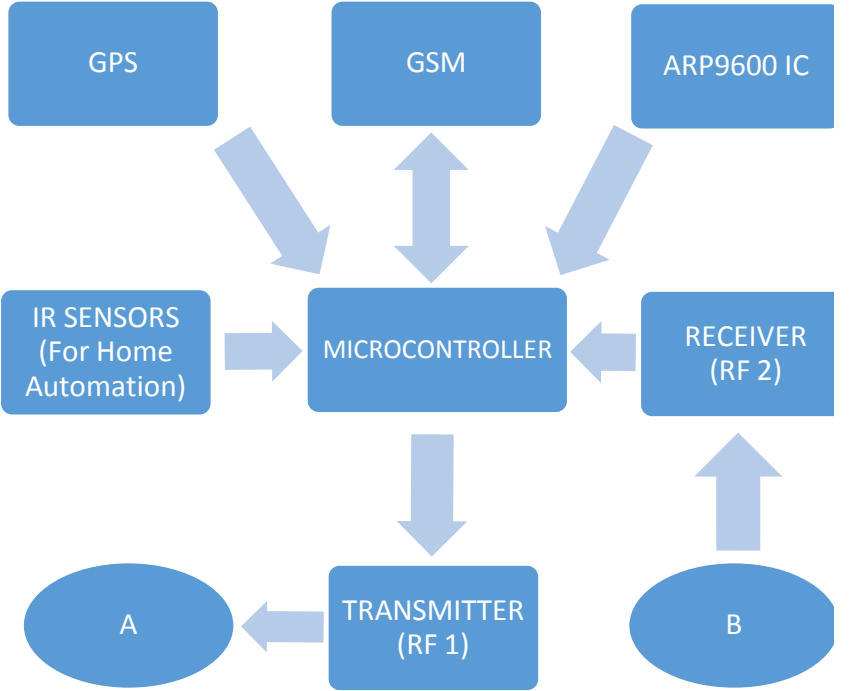

Figure 1: Block Diagram of Main Controlling Unit

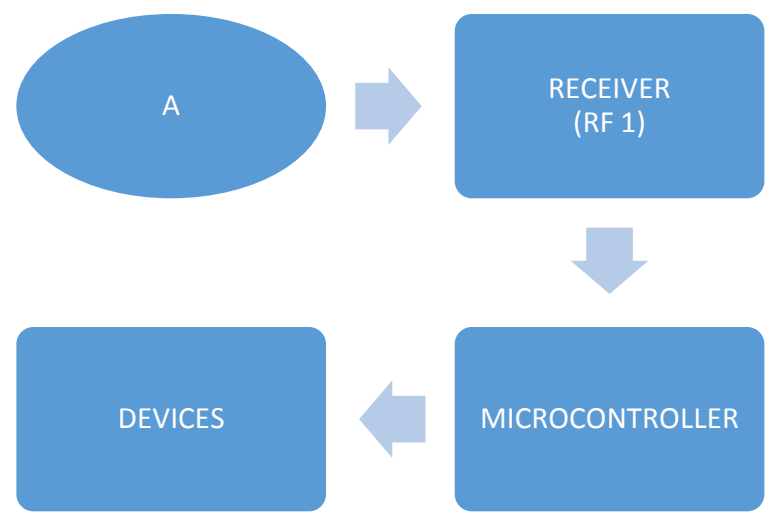

Figure 2: Block Diagram for Home Automation Feature

Security feature (block diagram shown in Figure 3) in the system is provided by the sensors: IR and Gas. When the Gas sensor detects smoke, any combustible gas or a value greater than its threshold value, it will get activated. The microcontroller will then activate GSM, GPS and buzzer to alert anyone nearby. Then the location information along with the text - "FIRE ALERT" will be issued via SMS alerts.

The IR sensor detects intrusion. It will be installed at the entrance. Any movement through the entrance will activate the IR. Then, through the RF signals, the microcontroller will be informed of the detection. Again following the same procedure the microcontroller will activate GSM, GPS and buzzer simultaneously. In this case, the GSM will issue SMS alerts containing the text - "INTRUDER ALERT" and location details.

Now, it may happen that the entry detected by the IR above is friendly or accidental. In this case, to avoid the activation of GSM, a key can be used. This key operates (on/off) the IR sensor installed at the entrance. When the IR sensor gets activated, the buzzer will sound alarm for five seconds initially. During this time interval if the user detects that the entry is friendly then, pressing the key will deactivate the buzzer and sensor. Otherwise, the microcontroller will activate the GSM, GPS and buzzer and the same procedure will follow.

Another key feature is the two way communication between the system and kin of the user. This will be provided through GSM. The kin will send an SMS with predefined text to the system's GSM. The SMS will request the data of the various sensors to check on the user. GSM will revert back to the request by providing the sensor details.

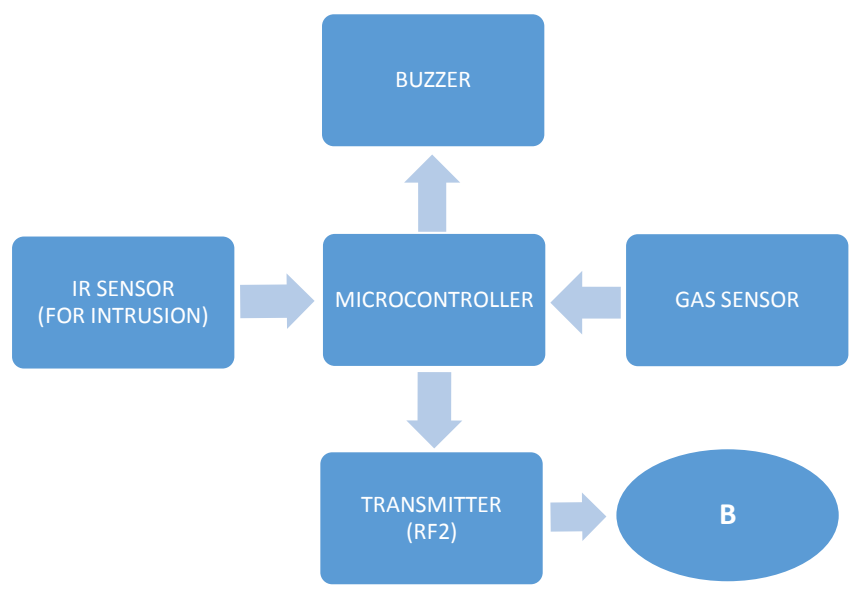

Figure 3: Block Diagram for Security Feature

\section{CONCLUSION}

There is no separate world for visually-impaired and so they have to live in the one with sighted people. Therefore, to make sure that they do not suffer due to their disability, they must be assisted in every way possible. An initiative in this direction, "All-in-one companion for visually-impaired" has been proposed to ensure safety and maintenance of basic needs of such people. Having said that, the system can also be modified so that it supports other important needs like - navigation, textto-speech output, FM Radio etc. Since it is considered difficult for a lonely visually-impaired to reach a destination, voice navigation system ${ }^{[11]}{ }^{[12]}$ can come handy. The map routes can be fed into the database and after entering the destination point, user is guided along the route through voice commands. Another modification possible is the development of a voice based platform that will interact with the user and carry out specific tasks given. For example, if an SMS is received on GSM Modem, the system will provide text-to-speech output and the user (visually-impaired) will be able to listen the content. Likewise if the user needs to send any message he/she can simply speak the content, recipient number and issue command 'SEND'. FM Radio for entertainment and time retrieval can also be included in the functions and accessed through commands like 'FM' and 'TIME' respectively. Thus, along with the safety and needs, system can be updated to provide a voice based platform and voice navigation system. Such modifications in the future will make the system more useful and beneficial for visually-impaired.

\section{REFERENCES}

[1] V Ramya, B Palaniappan, March 2012, "Embedded Home Automation for Visually Impaired", In International Journal of Computer Applications, Vol. 41, No. 18 Available at: $<$ http://www.ijcaonline.org/archives/volume41/number18/ $\underline{5643-8066>}$ 
[2]Wei Lun $\mathrm{Ng}$, Chee Kyun $\mathrm{Ng}$, Nor Kamariah Noordin, Borhanuddin Mohd. Ali, July 2011, "Gesture Based Automating Household Appliances", In Proceeding Part II of $14^{\text {th }}$ International Conference, HCI International, Vol. 6762, Pg. 285-293 Available at: $<$ http://link.springer.com/chapter/10.1007\%2F978-3-642$21605-3 \quad 32>$

[3] Yuze, H. Chaoqun Wang; Sugiura, S.; Otofuji, T.; Aikawa, T., March 2008, Presented in $22^{\text {nd }}$ International Conference on Advanced Information Networking and Application - Workshops, Pg. 38 - 43 Available at: $<$ http://ieeexplore.ieee.org/xpl/articleDetails.jsp?tp=\&arnu mber $=4482887 \& q u e r y T e x t \% 3 D v i s u a l l y+i m p a i r e d>$

[4] Takatori, N. Nojima, K; Matsumoto, M.; Yanashima, K.; Magatani, K., 2006, "Development of Voice Navigation System for the Visually Impaired by using IC tags", Presented In Engineering in Medicine and Biology Society, EMBS '06. $28^{\text {th }}$ Annual International Conference of the IEEE, Pg. $5181-5184$ Available at: $<$ http://ieeexplore.ieee.org/xpl/articleDetails.jsp?tp=\&arnu mber=4462971\&queryText\%3Dvisually+impaired $>$

[5] Balduzzi, L. Fusco, G.; Odone, F.; Dini, S.; Mesiti, M.; Destrero, A.; Lovato, A., September 2010, "Low Cost Face Biometry for Visually Impaired Users", Presented in 2010 IEEE Workshop on Biometric Measurements and Systems for Security and Medical Application, Pg. 45 - 52 Available $<$ http://ieeexplore.ieee.org/xpl/articleDetails.jsp?tp=\&arnu mber=5610444\&pageNumber\%3D2\%26queryText\%3Dvi sually+impaired>
[6] Carnegie Mellon Robotics Academy Available at: $<$ http://www.education.rec.ri.cmu.edu/content/electronics/ boe/ir sensor/1.html >

[7] MQ Gas Sensors Available at <http://playground.arduino.cc/Main/MQGasSensors>

[8] RF Module Available at 〈http://en.wikipedia.org/wiki/RF_module>

[9] APR9600 RE-Recording Voive IC - Single ChipVoice Recording \& Playback Device: 60-Second Duration Available 〈http://akizukidenshi.com/download/apr9600.pdf>

[10] Wireless Transmission through RF Module Available at: $<$ http://allaboutelectronics.hpage.in/wireless-transmissionthrough-rf-module_91868527.html >

[11] Jae Sung Cha, Dong Kyun Lim, Yong-Nyuo Shin, June 2013, "Design and Implementation of a Voice based Navigation for Visually Impaired Persons", In International Journal of Bio-Science and Bio-Technology, Vol. 5, No. 3, Pg. 61-68 Available at: < http://www.sersc.org/journals/IJBSBT/vol5_no3/6.pdf $>$

[12] V Ramya, Laxmi Raja, B Palaniappan, February 2013, "Voice Assisted Embedded Navigation System for the Visually Impaired", In International Journal of Computer Application, Vol. 64, No. 13 Available at: <http://www.ijcaonline.org/archives/volume64/number13/ 10697-5609> 